\title{
Closing the Gap: Characterizing Key Factors Leading to the Disparity in Suicide Rates along the Urban-Rural Continuum
}

\author{
Harrison Schurr, Andrei Tuluca ${ }^{*}$ and Beth Bailey \\ College of Medicine, Central Michigan University, Mount Pleasant, MI 48859, USA \\ *Corresponding Author: Andrei Tuluca. Email: tuluclam@cmich.edu
}

Received: 07 July 2021 Accepted: 17 September 2021

\begin{abstract}
Suicide is a top ten cause of mortality in the United States. In previous literature the suicide rates in rural communities have been reported to be greater than those of more urban communities. Additionally, these studies have discussed many potential causes for the unfortunate disparity in rates. One cause often discussed is lack of mental health care providers in rural communities. The data for this study was gathered from the CDC's WONDER database and the NPPES NPI Registry. The urban-rural categorization of counties used the 2013 NCHS Urban-Rural Scheme. Statistical analysis included chi-square tests, paired $t$-tests, and stepwise regression analyses. Results indicate that both the number of residents per provider $(r=0.35, p \leq 0.005)$, and urbanization level $(r=0.49$, $p \leq 0.001$ ) were significantly related to suicide rate. Additionally, even after controlling for provider rates, each additional level of rurality predicted an increase of 1.2 suicides per 100,000 residents. Ultimately, the number of providers may play a major role in suicide rates, but extra effort must also be made in rural communities to combat the other contextual factors leading to increased suicide rates.
\end{abstract}

\section{KEYWORDS}

Suicide; mental health care providers; rurality; mental health

\section{Introduction}

Suicide remains a top ten cause of mortality in the United States with a death rate of 13.9 per 100,000 people in 2019 [1]. Michigan, during the years 2015-2019, had a similar death rate at 13.8 per 100,000 . Interestingly, rates are extremely variable from county to county. These range from 33.9 in Alcona County, a rural county a great distance from any major city without any major employers, to 9.4 in Ottawa County, a county with a sizable proportion of upper-class suburban families outside of a major Metropolitan area [2]. Poverty, unemployment, education level, divorce rates, and deprivation all play major roles in determining suicide rates at the community level [3-5]. Further, the literature suggests rural counties suffer greater and more quickly rising death rates from suicide than their urban counterparts [6-10]. Number of providers, interpersonal isolation, workplace demands, and the values of rural populations all impact the rural vs. urban suicide rate disparity [11].

Further research is required to characterize the association more accurately between urbanization, number of healthcare professionals, and suicide rates. This study examines suicide rates, number of mental health care providers, and the urbanization of communities at the county level in the state of 
Michigan during the years 2015-2019. The authors of this study attempt to determine if more equitable distributions of mental health care professionals, or more focused attempts at addressing negative contextual factors, would produce higher efficacy when combating suicide rates. Understanding the effects that the number of mental healthcare professionals has on a specific community based on its 2013 NCHS (National Center for Health Statistics) Urban-Rural Classification Scheme will allow for the more productive utilization of resources in the battle against suicide.

We expect to see that more rural locations will have less mental health providers and subsequently significantly higher suicide rates. It is further anticipated to see a paradigm in which rural communities will continue to demonstrate increased suicide rates due to negative contextual factors even after controlling for the number of providers. This is the first retrospective study to analyze data within the state of Michigan for suicide rates, mental health care providers, and the potential strain on mental health care resources that exists today.

\section{Methods}

All suicide rates for this study were obtained using the CDC's WONDER database [12]. The underlying cause of death data available on WONDER are county-level national mortality and population data spanning the years 1999-2019. The data, which is freely available to the public, is based on the death certificates for U.S. residents. Each one of these certificates denotes the demographic data of the person it is representing as well as a single underlying cause of death. The input specifications for WONDER this study uses are all the counties in Michigan, county codes 26001-26165. Demographics specified included all ages, races, origins, and genders. Years scrutinized were 2015 to 2019. All weekdays, all autopsy values, and all locations of death were included. ICD-10 codes of interest were X60-X84 (Intentional Self Harm).

The urbanization of each of these counties was determined using the 2013 NCHS Urban-Rural Scheme for Counties. Counties are classified as: Large Central Metro, Large Fringe Metro, Medium Metro, Small Metro, Micropolitan (non-metro), and Noncore (non-metro). The large central metro category contains counties in metropolitan statistical areas (MSAs) of one million or more population that have been identified by NCHS classification rules as central because they contain all or part of a principal city of the area. The large fringe metro category contains the remaining counties (similar to suburbs) in MSAs of one million or more. Counties in MSAs of 250,000 to 999,999 population are assigned to the medium metro category and counties in MSAs with populations under 250,000 are assigned to the small metro category. Nonmetropolitan counties that are designated by the Office of Management and Budget as belonging to a micropolitan statistical area are assigned to the micropolitan category and the remaining nonmetropolitan counties are assigned to the noncore category. The large central metro category is the most "urban" category, and the noncore category is the most "rural" category [13].

An additional database used was the National Provider Identifier (NPI) records. The NPI Registry enables you to search for a provider's National Plan and Provider Enumeration System (NPPES) information. All information produced by the NPI Registry is provided in accordance with the NPPES Data Dissemination Notice. Information in the NPI Registry is updated daily. Simple queries can be run to retrieve this read-only data. The data for this study was retrieved from this registry using County Health Rankings \& Roadmaps, a program of the University of Wisconsin Population Health Institute [14]. This program reports county-specific numbers of mental health care providers. Specifications for the query for this study included all counties in the state of Michigan in 2017 and mental health care providers. Mental health providers are defined as psychiatrists, psychologists, licensed clinical social workers, counselors, marriage and family therapists, and mental health providers that treat alcohol and other drug abuse, as well as advanced practice nurses specializing in mental health care.

Statistical analysis conducted for this study included chi-square tests, paired $t$-tests, and stepwise regression analyses to examine the links among the study variables. 


\section{Results}

\subsection{County Inclusion}

The retrieved WONDER data set contained complete urbanization code and provider number information for 76 of the 83 Michigan counties. Suicide rate was missing/unstable due to small sample size for an additional 14 counties, so the final analyses were performed for 62 Michigan counties. Table 1 demonstrates the percentage of counties included in this study according to their urbanization code and category. As shown, Micropolitan and Non-Core County classifications were the most popular counties in Michigan (Table 1).

Table 1: Characteristics of michigan counties included $(n=62)$

\begin{tabular}{ll}
\hline & Mean or rate \\
\hline Number of residents per provider & $752(210-3720)$ \\
Urbanization code & \\
Large central or fringe metro (code $=1$ or 2$)$ & $16.1 \%$ \\
Medium metro $($ code $=3)$ & $12.9 \%$ \\
Small metro $($ code $=4)$ & $12.9 \%$ \\
Micropolitan $($ code $=5)$ & $30.6 \%$ \\
Non-core $($ code $=6)$ & $27.4 \%$ \\
Crude suicide rate per 100,000 & $17.8(9.6-27.7)$ \\
\hline
\end{tabular}

\subsection{Suicide Rates per County Urbanization Level}

Both number of residents per provider $(\mathrm{r}=0.35, p \leq 0.005)$, and urbanization level $(\mathrm{r}=0.49, p \leq 0.001)$ were significantly related to suicide rate, with more residents per provider and decreasing levels of urbanization predicting higher suicide rates. An examination of suicide rates by categorical urbanization level also revealed a significant association $\left(\chi^{2}=282.1, p=0.001\right)$, with rates increasing as rurality increased (Table 2).

Table 2: Suicide rates by county urbanization code

\begin{tabular}{ll}
\hline Urbanization level & Suicide rate $($ per 100,000) \\
\hline Large central or fringe metro $($ code $=1$ or 2$)$ & 15.1 \\
Medium metro $(\operatorname{code}=3)$ & 15.6 \\
Small metro $(\operatorname{code}=4)$ & 16.4 \\
Micropolitan $($ code $=5)$ & 17.9 \\
Non-core $($ code $=6)$ & 20.8 \\
\hline
\end{tabular}

\subsection{Residents per Provider, Urbanization Level, and Suicide Rate}

Whether urbanization predicted suicide rates beyond the effect of rates of providers in counties was analyzed. A regression analysis was performed, entering number of residents per provider on the first step, followed by entry of urbanization level on the second step. Results indicate that even after control for provider rates, each additional level of rurality on the NCHS Urban-Rural Scheme for Counties predicted an increase of 1.2 suicides per 100,000 residents (Table 3). Additionally, both factors together accounted for $28 \%\left(\mathrm{R}^{2}=0.281\right)$ of the variance in suicide rates. Finally, after controlling for level of 
urbanization, each additional 500 residents per provider predicted an increase of 1 additional suicide per 100,000 residents (Table 3).

Table 3: Stepwise linear regression results predicting county suicide rates

\begin{tabular}{lllllll}
\hline & $\begin{array}{l}\text { Unstandardized } \\
\text { regression } \\
\text { coefficient (b) }\end{array}$ & $\begin{array}{l}\text { Standardized } \\
\text { regression } \\
\text { coefficient }(\beta)\end{array}$ & t & $p$ & $\begin{array}{l}\mathrm{R}^{2} \\
\text { change }\end{array}$ & $p$ \\
\hline $\begin{array}{l}\text { Step 1 } \\
\text { Residents per provider }\end{array}$ & 0.003 & 0.350 & 2.89 & 0.005 & & \\
Step 2 & & & & & 0.16 & 0.001 \\
Residents per provider & 0.002 & 0.201 & 1.70 & 0.094 & & \\
Urbanization level & 1.208 & 0.423 & 3.58 & 0.001 & & \\
\hline
\end{tabular}

\section{Discussion}

Suicide is a financially straining and preventable cause of death in the United States. In 2013 alone, the national cost of reported suicide and suicidal attempts was $\$ 58.4$ billion dollars [15]. After adjusting for a variety of parameters, Shepard et al. [15] demonstrated an annual public cost of $\$ 93.5$ billion dollars. This cost is initially staggering; however, it becomes more understandable when considered in the context of the resources it funds. Notably, the National Suicide Prevention Lifeline, a public phone number which provides free services available $24 \mathrm{~h}$ a day, seven days a week, and the Crisis Text Line, a messaging service which allows individuals to communicate via text with appropriate personnel. These two resources alone obviously do not add up to Shepard et al.'s [15] \$93.5 billion dollars, but a simple web search about suicide prevention will immediately produce information on thousands of other public resources, societies that educate their readers on the warning signs of suicide, and hotlines. Furthermore, typical provider visits with Primary Care Physicians elicit questions regarding depression, anxiety, and suicidal thoughts and behaviors through population screening questionnaires such as the Patient Health Questionnaire-9. Beyond the financial cost, there is also a tremendous psychological, emotional, and physical toll that suicide and suicidal attempts take on both the individual committing these acts, as well as those involved in the individual's life.

Despite all these resources and strategies to catch potentially suicidal patients, suicide rates have increased nearly 30\% from 2000 to 2016 alone [16]. Additionally, it has been documented previously that suicide rates are increasing in Michigan [17]. When breaking down further, some research has attributed this increase in suicide rates to the ongoing public health crisis of opioid deaths [18]. While a plethora of data exists tying suicide to a variety of causes, of particular focus within this study was its relationship to the level of urbanization of a county as well as the number of mental health providers within the state of Michigan.

Furthermore, with roughly a fifth of Michigan's population living in rural communities, it is important to recognize the faster rise of suicide in rural communities in the US [19]. Our study demonstrated that the less urban (and therefore more rural) the county in Michigan, the higher the rate of suicide. This aligns with previous studies and theory outlining factors that lead to increased suicide in rural counties. These factors include, but are not limited to, social isolation, higher rates of interpersonal violence, higher access to firearms, and reduced access to mental health care both due to resource deficits and perceived stigma [20]. Of note is the stigma regarding mental health, suicide and help seeking that predominates in the rural health culture. Interestingly, Steward et al. [21] demonstrated that when controlling for level of 
education, employment, and income, older adults in rural counties were more likely to have elevated levels of public and self-stigma and less tolerance of psychological openness when compared to their urban counterparts. In another study by Monteith et al. [22], the relationship between stigmas towards suicide and suicide rate for veterans in rural communities was explored. After surveying individuals, results demonstrated that suicide related stigma may in fact be one of the most important things to prioritize in deconstructing as a community-based suicide prevention program in rural communities to decrease suicide rates in veterans in rural counties [22]. This speaks to the potential downfalls of many suicide prevention constructs in place today which rely heavily on technology and access to either said technology or those who possess it. This could potentially account for the increasing rates of suicide in rural communities in Michigan and the noted relative higher rates of suicide in Michigan's rural counties when compared to the more urbanized ones.

Another potential factor that may account for the significantly higher rates of suicide seen in rural counties in Michigan when compared to more urban counties could be explained by the distrust in the health care system in rural counties. Additionally, the distrust in the health care system could attribute to the fact that $45 \%$ of people who commit suicide will have seen their primary care provider within the month before their death and only $20 \%$ will have seen a mental health professional [23]. It is quite possible that patients with thoughts of suicide did not follow the treatment plan of their primary care provider due to a mistrust in the provider's judgment. Further, it may be the case that the patient did not bring up their suicidal ideation due to a belief that the primary care physician could not or would not be willing to help them in that aspect of health. With many of those who committed suicide seeing only their primary care physician it is clear that a more robust and effective referral process must be implemented. However, this strategy, at least for the time being, may not be the most effective due to the inaccessibility of mental health care providers even in urban areas. A study out of Vancouver, Canada demonstrated a referral success rate of $3 \%$, represented by 6 of the 230 psychiatrists contacted. In addition to poor referral success rate, average wait times ranged from 4 to 55 days [24]. This type of wait time and inaccessibility would vastly diminishes any effect a more complete screening process would have and necessitates further research and initiatives to fix. Jones et al. [25] demonstrated that in the context of depression treatments, a contributing factor to suicidal behaviors, urban patients were more likely to agree and heed advice of healthcare professionals regarding treatment when compared to their rural counterparts. This may account for the findings of our study which demonstrated that even with control for provider rate, rural counties in Michigan demonstrated an increase rate of suicide relative to their more urban county counterparts.

This begs the question regarding the utilization of mental health providers in counties with less urbanization. The results of this study showed, in congruence with the studies discussed previously, a statistically significant increase in suicide rates with increasing rurality $(\mathrm{r}=0.49, p<0.001)$. In addition, a significant correlation between mental health care providers and suicide rates, with each additional 500 patients per provider predicting an increase of 1 additional suicide per 100,000 residents was demonstrated. More interestingly, this novel study numerically demonstrates that rural communities in Michigan have higher suicide rates than urban communities even when controlling for the number of mental health care providers. This finding is not well documented in the literature. While in many communities the lack of providers may be a significant issue, this study has shown that attributing the difference in suicide rates between urban and rural communities only to an inadequate number of providers in incorrect. It is important to note here that although this study focuses on Michigan and its counties, the results do not necessarily pertain to these communities. The results, by merit of using a national scheming mechanism to determine the rurality of an area, are potentially applicable to other communities in the US, if going off the 2013 NCHS (National Center for Health Statistics) Urban-Rural Scheme for Counties. 
Unique to this paper is the understanding that rural communities require more providers per capita to obtain similar suicide rates as urban communities. This finding reveals two potential avenues for the utilization of resources and providers in the fight against suicide in the USA. The first, is to provide incentives for mental health care providers to work in rural areas. Unfortunately, with the already staggering nationwide shortage of mental health providers, shifting providers to rural communities will only leave urban communities at larger scarcities, creating an unacceptable trade off [26]. It may be the case however, that the mental health care provider shift from urban to rural does not have to be a physical one. Over the last few years there has been an increase in online services directed towards bettering the mental health and wellbeing of the user. This may allow rural populations to gain access to care and resources not accessible to them previously. Examples of these types of services include ChatPAL, Cerebral, and BetterHelp. ChatPAL is a project headed by Ulster University that aims to develop and trial a chatbot to support and promote mental wellbeing in rural Europe. Cerebral and BetterHelp are online based services designed to remotely connect patients to mental health care providers without the need for in-person appointments. While there are limited studies characterizing the efficacy of these services, preliminary studies have shown that cognitive behavioral therapy and acceptance and commitment therapy delivered virtually are beneficial in patients requiring intervention [27]. Additionally, it has been shown that an adequate therapeutic alliance can be formed virtually, suggesting that it may be possible for any speech-based therapy to be delivered through such a medium [28]. Although promising, further investigations into the efficacy of virtual treatment must be made before it can become a definitive tool in the fight against rural suicide.

The second potential avenue is to focus on interventions aimed at reducing suicide rates in ways independent of total number mental health providers, such as interventions that deconstruct the various cultural and social norms that prevail in more rural communities. Addressing the previously identified key components in rural suicide rates such distrust in health care providers, stigma against mental health issues, and suicidal behaviors appears to the authors of this study to be the most impactful course of action that can be taken to change the current worsening trends in suicide rates. There exist several approaches that have demonstrated efficacy in addressing these components. For example, a social marketing campaign in Murrumbidgee, Australia was able to utilize multiple social media platforms along with local insights and approaches to increase awareness of services, reduce stigma and encourage helpseeking at a time of crisis [29]. Ideally this type of campaign could, with alterations making it specific to the community of interest, be implemented in rural communities suffering from high rates of suicide to increase the use of resources already in place. A study on effective research recruitment and preventative intervention demonstrated members of a community prefer to interact with someone who matches their cultural background [30]. The study also mentioned that the use of a well-respected and connected community liaison was critical to the success of recruitment. The use of this liaison, as well as recruitment at community events, referrals from community agencies and organizations, and the use of social medial lead to an significant increase in participation and fostered a new trust between the researches and community. While not directly related to suicide prevention, this type of intervention could be applied to suicide awareness and prevention with little difficulty. The study as a whole suggests that utilizing well-respected members of the local community, local businesses and organizations, and social media to combat the distrust in mental health care providers and stigma against mental health issues in a rural community would be an effective plan of action. In addition to this, access to firearms and its relationship to suicide completion should not be ignored and addressed, although the authors feel as though this transcends a healthcare issue due to the political implications of gun laws in America. 


\section{Limitations}

Although there are many strengths to this study, there are limitations as well, some of which are inherent to database studies themselves. First, this study, being an ecological study, does not allow for interpretation of data on the individual level. Rather, it analyzes data on the county level. Second, it is possible for major rurality variation within counties to be present and not accounted for in the overall urbanization of the county. Third, not all suicides are recorded as such in the national mortality data, leading to possible underestimation. Fourth, not all people who died by suicide necessarily had previous mental health concerns requiring psychiatric or psychologic treatment. If the number of suicides of this type was significant, it could potentially create an elevation in suicide rate that is irrespective of urbanicity and cannot be combated by traditional means. However, with the data gathered from WONDER, it is impossible to determine the number of suicides of this type. Fifth, while rurality was determined using a scheme applicable to any county in the USA, the exact contextual factors influencing suicide in the State of Michigan may not be equivalent to those influencing other areas of the USA. Although, they could just as easily be the same as different. Sixth, not all providers have a National Provider Identification number, some National Provider Identification numbers belong to providers no longer practicing or accepting new patients, and some National Provider Identification numbers belong to providers practicing in different counties than their number suggests. These discrepancies could lead to artificially inflated or deflated number of providers in a specific community depending on the circumstances. Finally, it is possible for confounding variables not controlled for are altering analyses. These include, but are not limited to, the closing of a major cooperation that previously employed most of the community's population, a particularly harsh growing season leading to a significant decrease in income for farmers, or civil unrest and social injustices within a metropolitan area. All these occurrences have the potential to drastically increase suicide rates in a community without being accounted for in the final analyses.

\section{Conclusion}

This study examined suicide rates and number of mental health care professionals by urbanization on a county level in the State of Michigan. Suicide rates were shown to increase with both the increasing rurality of a community as well as with a decrease in the number of providers. Most importantly, this study demonstrated that even if the number of providers per capita were equal, rural communities would still experience higher rates in suicide than their more urban counterparts. These findings suggest that in addition to increasing providers, increased efforts and resources directed at combating contextual factors such as distrust in health care providers, stigma against mental health issues, and suicidal behaviors in rural communities should be taken [31]. Collectively, these implementations would be a more cost effective and efficient way to oppose rising suicide rates instead of continuing ongoing trends of shifting an already inadequate number of mental health care providers.

Acknowledgement: There are no other contributors who meet the criteria for authorship.

Funding Statement: The authors received no specific funding for this study.

Conflicts of Interest: The authors declare that they have no conflicts of interest to report regarding the present study.

\section{References}

1. Kochanek, K. D., Xu, J. Q., Arias, E. (2020). Mortality in the United States, 2019. NCHS Data Brief, No. 395. Hyattsville, MD: National Center for Health Statistics.

2. Centers for Disease Control and Prevention, National Center for Health Statistics (2021). Underlying Cause of Death 1999-2019 on CDC WONDER Online Database, released in 2020. Data are from the Multiple Cause of 
Death Files, 1999-2019, as compiled from data provided by the 57 vital statistics jurisdictions through the Vital Statistics Cooperative Program. http://wonder.cdc.gov/ucd-icd10.html.

3. Rehkopf, D. H., Buka, S. L. (2006). The association between suicide and the socio-economic characteristics of geographical areas: A systematic review. Psychological Medicine, 36(2), 145-57. DOI 10.1017/S003329170500588X.

4. Milner, A., Page, A., LaMontagne, A. D. (2014). Cause and effect in studies on unemployment, mental health and suicide: A meta-analytic and conceptual review. Psychological Medicine, 44(5), 909-917. DOI 10.1017/ S0033291713001621.

5. Chen, J., Choi, Y. J., Mori, K., Sawada, Y., Sugano, S. (2012). Socio-economic studies on suicide: A survey. Journal of Economic Surveys, 26(2), 271-306. DOI 10.1111/j.1467-6419.2010.00645.x.

6. Ivey-Stephenson, A. Z., Crosby, A. E., Jack, S. P., Haileyesus, T., Kresnow-Sedacca, M. (2017). Suicide trends among and within urbanization levels by Sex, race/Ethnicity, age group, and mechanism of death. Surveill Summ, 66(18), 1-16.

7. Rossen, L. M., Hedegaard, H., Khan, D., Warner, M. (2018). County-level trends in suicide rates in the U.S., 20052015. American Journal of Preventive Medicine, 55(1), 72-79. DOI 10.1016/j.amepre.2018.03.020.

8. Kegler, S. R., Stone, D. M., Holland, K. M. (2017). Trends in suicide by level of urbanization. Morbidity and Mortality Weekly Report, 66(10), 270-273. DOI 10.15585/mmwr.mm6610a2.

9. Fontanella, C. A., Hiance-Steelesmith, D. L., Phillips, G. S. (2015). Widening rural-urban disparities in youth suicides. JAMA Pediatric, 169(5), 466-473. DOI 10.1001/jamapediatrics.2014.3561.

10. Singh, G. K., Siahpush, M. (2002). Increasing rural-urban gradients in US suicide mortality, 1970-1997. American Journal of Public Health, 92(7), 1161-1167. DOI 10.2105/ajph.92.7.1161.

11. Hirsch, J. K., Cukrowicz, K. C. (2014). Suicide in rural areas: An updated review of the literature. Journal of Rural Mental Health, 38(2), 65-78. DOI 10.1037/rmh0000018.

12. "CDC Wonder" Centers for Disease Control and Prevention, Centers for Disease Control and Prevention. https:// wonder.cdc.gov/.

13. Ingram, D., and Shelia F. (2015). Centers for Disease Control and Prevention. https:/www.cdc.gov/nchs/data/ series/sr_02/sr02_166.pdf.

14. https://www.countyhealthrankings.org/app/michigan/2018/measure/factors/62/data.

15. Shepard, D. S., Gurewich, D., Lwin, A. K., Reed, G. A. Jr, Silverman, M. M. (2016). Suicide and suicidal attempts in the United States: Costs and policy implications. Suicide \& Life-Threatening Behavior, 46(3), 352-362. DOI 10.1111/sltb.12225.

16. Nugent, A. C., Ballard, E. D., Park, L. T. (2019). Research on the pathophysiology, treatment, and prevention of suicide: Practical and ethical issues. BMC Psychiatry, 19, 332. DOI 10.1186/s12888-019-2301-6.

17. https://www.americashealthrankings.org/explore/annual/measure/Suicide/state/MI.

18. Segal, L. M., de Biasi, A., Mueller, J. L., May, K. et al. (2017). Pain in the nation: The drug, alcohol, and suicide crises and the need for a national resilience strategy. Pain in the Nation: Public Health Report. Oakland: Well Being Trust.

19. “About NCHS-Homepage" (2020). Centers for Disease Control and Prevention. https://www.cdc.gov/nchs/ about/index.htm.

20. Monteith, L. L., Holliday, R., Brown, T. L., Brenner, L. A., Mohatt, N. V. (2021). Preventing suicide in rural communities during the COVID-19 pandemic. The Journal of Rural Health, 37(1), 179-184. DOI 10.1111/ jrh.12448.

21. Stewart, H., Jameson, J. P., Curtin, L. (2015). The relationship between stigma and self-reported willingness to use mental health services among rural and urban older adults. Psychological Services, 12(2), 141-148. DOI 10.1037/ a0038651.

22. Monteith, L. L., Smith, N. B., Holliday, R., Dorsey, H., Brooke, A. et al. (2020). We're afraid to say suicide. The Journal of Nervous and Mental Disease, 208(5), 371-376. DOI 10.1097/NMD.0000000000001139. 
23. Luoma, J. B., Martin, C. E., Pearson, J. L. (2002). Contact with mental health and primary care providers before suicide: A review of the evidence. The American Journal of Psychiatry, 159(6), 909-916. DOI 10.1176/appi. ajp.159.6.909.

24. Goldner, E. M., Jones, W., Fang, M. L. (2011). Access to and waiting time for psychiatrist services in a Canadian urban area: A study in real time. The Canadian Journal of Psychiatry, 56(8), 474-80. DOI 10.1177/ 070674371105600805 .

25. Jones, A. R., Cook, T. M., Wang, J. (2011). Rural-urban differences in stigma against depression and agreement with health professionals about treatment. Journal of Affective Disorders, 134(1-3), 145-150. DOI 10.1016/j. jad.2011.05.013.

26. US Department of Health and Human Services (2016). "National projections of supply and demand for selected behavioral health practitioners: 2013-2025." Rockvile, Maryland. https://bhw.hrsa.gov/sites/default/files/bhw/ healthworkforceanalysis/research/projections/behavioral-health2013-2025.pdf.

27. Barrett, K., Stewart, I. (2021). A preliminary comparison of the efficacy of online acceptance and commitment therapy (ACT) and cognitive behavioural therapy (CBT) stress management interventions for social and healthcare workers. Health \& Social Care in the Community, 29, 113-126. DOI 10.1111/hsc.13074.

28. Jonathan, E. (2002). Cook and carol doyle. Cyber Psychology \& Behavior, 5(2), 95-105. DOI 10.1089/ 109493102753770480 .

29. Loton, C., Neal, M. (2021). Mental health, alcohol and other drugs: After hours crisis marketing initiative, a unique and local social marketing campaign within a regional and rural Australian setting. In: Hay, R., Eagle, L., Bhati, A. (Eds.), Broadening cultural horizons in social marketing. Springer, Singapore. DOI 10.1007/978-981-15-8517-3_7.

30. Murry, V. M., Brody, G. H. (2004). Partnering with community stakeholders: Engaging rural african American families in basic research and the strong african American families preventive intervention program. Journal of Marital and Family Therapy, 30(3), 271-283. DOI 10.1111/j.1752-0606.2004.tb01240.x.

31. Steelesmith, D. L., Fontanella, C. A., Campo, J. V., Bridge, J. A., Warren, K. L. et al. (2019). Contextual factors associated with county-level suicide rates in the United States, 1999 to 2016. JAMA Network Open, 2(9), e1910936. DOI 10.1001/jamanetworkopen.2019.10936. 